\title{
Laparoscopic splenectomy for immune thrombocytopenia in patients with a very low platelet count
}

\author{
Anna Zychowicz ${ }^{1}$, Dorota Radkowiak ${ }^{1}$, Anna Lasek ${ }^{1}$, Piotr Małczak ${ }^{1,2}$, Jan Witowski ${ }^{1,2}$, Piotr Major ${ }^{1,2}$, Marcin Strzałka ${ }^{1}$, \\ Jan Kulawik ${ }^{1}$, Andrzej Budzyński ${ }^{1,2}$, Michał Pędziwiatr ${ }^{1,2}$ \\ ${ }^{1} 2^{\text {nd }}$ Department of General Surgery, Jagiellonian University Medical College, Krakow, Poland \\ ${ }^{2}$ Centre for Research, Training and Innovation in Surgery, Krakow, Poland
}

Videosurgery Miniinv 2018; 13 (2): 157-163

DOI: https://doi.org/10.5114/wiitm.2018.75847

\begin{abstract}
Introduction: Laparoscopic splenectomy (LS) is the gold standard in treating immune thrombocytopenia (ITP). However, there are still some problems in decision-making when considering LS in patients with a very low platelet count (PLT). Aim: To evaluate safety outcomes of LS in patients with severe ITP and very low PLT in comparison to those with higher PLT.

Material and methods: We retrospectively analyzed consecutive patients who underwent LS in a single institution between April 1998 and December 2017. Perioperative care was based on an algorithm developed at our department which takes into consideration the patient's PLT level. Patients were divided into 2 groups depending on the PLT level (cut-off point 50,000/ $\mathrm{mm}^{3}$ ).

Results: The mean operative time in the low PLT group and high PLT group was $90 \pm 42.1$ min and $95 \pm 45 \mathrm{~min}$, respectively $(p=0.59)$. Intraoperative blood loss was $144 \pm 226.1 \mathrm{ml}$ in the low PLT group and $83 \pm 161.24 \mathrm{ml}$ in the high PLT group $(p=0.23)$. Complications occurred in $5(9.09 \%)$ patients in the low PLT group and $16(11.51 \%)$ in the high PLT group $(p=0.67)$. There were no conversions in the group with lower PLT, while 2 patients in the group with higher PLT had to be converted to open surgery $(p=0.38)$. Patients with low PLT preoperatively more often required perioperative platelet transfusions (13 vs. $1, p<0.001$ ).

Conclusions: Laparoscopic splenectomy is safe and feasible treatment in patients with ITP regardless of the PLT level. Still, patients with critical ITP and marginally low PLT require special awareness.
\end{abstract}

Key words: laparoscopy, splenectomy, immune thrombocytopenia.

\section{Introduction}

Intra- and postoperative bleeding is one of the more common surgical complications. Its occurrence depends mostly on the type and the extent of the procedure as well as coagulation profile of the patient. In fact, any hematological disorder related to coagulopathy is by definition associated with increased risk of perioperative bleeding. Immune thrombocy- topenia (ITP), also known as idiopathic or immune thrombocytopenic purpura, is a relatively common hematologic disease characterized by immune-mediated destruction of platelets, mostly in the spleen, leading to a low platelet count (PLT) and bleeding [1]. Among treatment modalities splenectomy is considered a main second-line treatment in refractory cases that do not respond to conservative management. In 1991, the very first laparoscopic splenecto-

Address for correspondence

Michat Pędziwiatr MD, PhD, $2^{\text {nd }}$ Department of General Surgery, Jagiellonian University Medical College; Centre for Research,

Training and Innovation in Surgery (CERTAIN Surgery), 21 Kopernika St, 31-501 Krakow, Poland, phone: +48 6085523 23,

e-mail: michal.pedziwiatr@uj.edu.pl 
my (LS) was conducted by Delaitre and Maignien [2]. Since then LS has become the gold standard for patients suffering from ITP and has gradually replaced traditional open splenectomy (OS) [3]. Laparoscopic splenectomy has many benefits such as lower rates of morbidity and mortality, reduced surgical trauma and blood loss, lower postoperative pain, better cosmetic appearance and shorter hospital stay [4]. Uncorrected coagulopathy is considered an absolute contraindication to surgical procedures in general and is particularly strongly emphasized in the case of laparoscopy $[5,6]$. There are potential problems in decision-making when considering LS in severe cases of ITP with extreme low PLT. It is commonly accepted that the safe PLT count to perform surgery is $50,000 / \mathrm{mm}^{3}$, but in patients with ITP it is not always possible to achieve [4]. Therefore, in this study we aimed to evaluate the safety of LS in patients with severe thrombocytopenia.

\section{Aim}

The aim of the study was to evaluate safety outcomes of laparoscopic splenectomy in patients with severe ITP and very low PLT in comparison to those with higher PLT.

\section{Material and methods}

Consecutive patients undergoing laparoscopic splenectomy for ITP in a university tertiary referral center between April 1998 and December 2017 were included in the study. Before surgery, all patients underwent preoperative assessment and were treated conservatively by a consultant hematologist in order to control the disease and increase platelet levels. In the case of treatment failure or complications related to used medications, patients underwent elec- tive laparoscopic splenectomy despite low PLT. Decision-making about whether to perform an LS was assessed using our internal algorithm. Patients were preoperatively managed depending on the PLT level (Figure 1):

1. Patients with PLT $\geq 50,000 / \mathrm{mm}^{3}$ were selected for laparoscopic surgery without any additional preoperative means.

2. Patients with PLT $<50,000 / \mathrm{mm}^{3}$ were reevaluated by hematologists and an attempt to raise platelets to the level $\geq 50,000 / \mathrm{mm}^{3}$ was undertaken. If successful, patients were treated by LS.

3. Patients in whom the hematologist were able to achieve only a partial response and whose preoperative PLT were between $20,000 / \mathrm{mm}^{3}$ and $50,000 / \mathrm{mm}^{3}$ were scheduled for LS after ensuring that the blood bank could deliver platelet transfusions immediately whenever needed. Most of them however did not require transfusions.

4. Patients with preoperative PLT $<20,000 / \mathrm{mm}^{3}$ despite intensive preoperative conditioning were operated on and platelet transfusions were administered intraoperatively in order to elevate PLT level.

The operative method of choice in our department is a laparoscopic 4-port splenectomy, which was performed as described elsewhere [7]. In some patients single incision splenectomy was performed [8]. None of the patients with ITP was initially operated on with an open approach. In all cases routine hematological treatment was implemented before surgical intervention, and the indications for splenectomy included failure of conservative treatment or side-effects of drugs used that did not allow further therapy. Preoperatively, platelet level was routinely measured within $8 \mathrm{~h}$ prior to surgery. For the purpose of statistical analyses patients with preoperative PLT

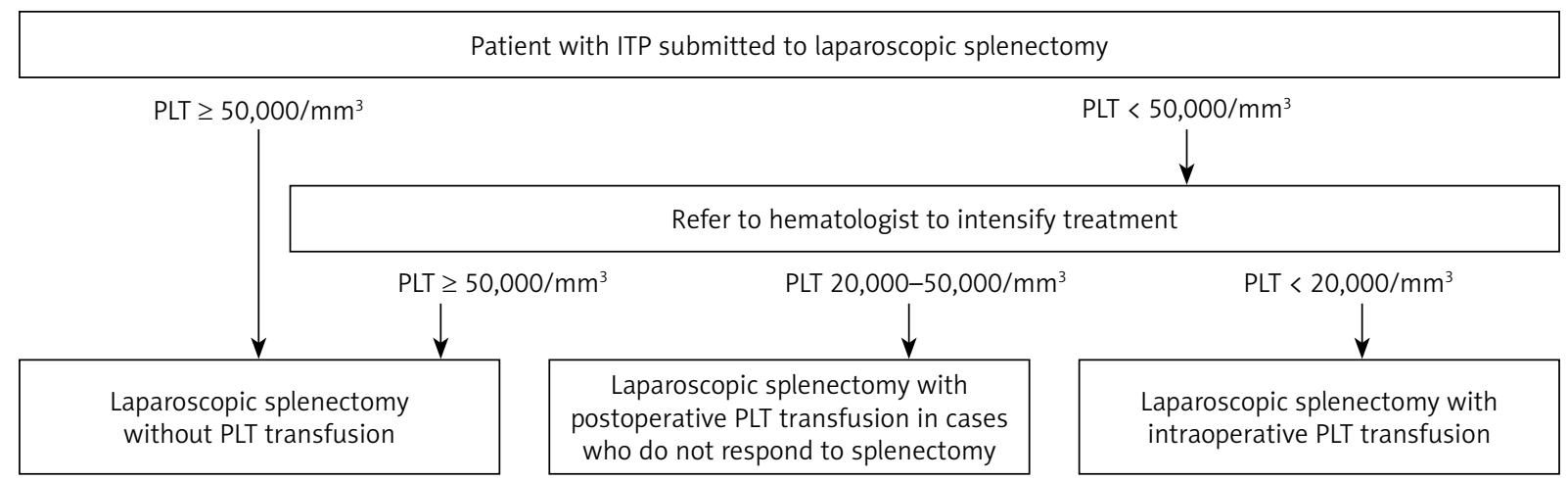

Figure 1. Decision-making algorithm in patients with ITP who underwent laparoscopic splenectomy 
$<50,000 / \mathrm{mm}^{3}$ (low PLT group) were compared with those with PLT $\geq 50,000 / \mathrm{mm}^{3}$ (high PLT group).

The measured outcomes were the operative time, intraoperative blood loss, need for blood transfusions, conversion rate and morbidity. Operative time was measured from skin incision to closure. The intraoperative blood loss was measured from the amount of blood aspirated in the suction machine. The study was conducted according to STROBE guidelines [9]. All procedures followed the ethical standards of the responsible committee on human experimentation (institutional and national) and the sixth revision (Fortaleza) of the 1975 Declaration of Helsinki. The study was approved by an independent ethics committee of the Jagiellonian University, Krakow, Poland (approval number 1072/6120/160/2017). Informed consent for the surgical treatment was obtained from all patients before the procedure.

\section{Patients}

Out of 543 patients undergoing laparoscopic splenectomy in our department in the study period,
194 were operated on for ITP. Group 1 consisted of 9 patients with PLT $<20,000 / \mathrm{mm}^{3}$. group 2 consisted of 46 patients with PLT $\geq 20,000 / \mathrm{mm}^{3}$ and PLT $<50,000 / \mathrm{mm}^{3}$. Group 3 comprised 114 patients with initial PLT $<50,000 / \mathrm{mm}^{3}$ that was elevated to at least $50,000 / \mathrm{mm}^{3}$ preoperatively, and group 4 included 25 patients with PLT $\geq 50,000 / \mathrm{mm}^{3}$. Patients' demographic characteristics are presented in Table I. For the purpose of statistical analyses patients in groups 1 and 2 were combined as the low PLT group and analyzed together with groups 3 and 4 (high PLT group). There were no significant differences in demographics between analyzed groups (Table I).

\section{Statistical analysis}

Statistical analysis was performed using StatSoft Statistica version 13.0 software. Normal distribution of continuous variables was tested with the $\chi^{2}$ test. Variables with non-normal distribution were compared using the Mann-Whitney $U$ test. Categorical variables were compared with the $\chi^{2}$ test. Results

Table I. Demographic data of patients

\begin{tabular}{|c|c|c|c|c|c|}
\hline \multirow[t]{2}{*}{ Parameter } & $\begin{array}{c}\text { Group } 1 \\
\left(\mathrm{PLT}<20,000 / \mathrm{mm}^{3}\right)\end{array}$ & $\begin{array}{c}\text { Group 2 } \\
\left(\text { PLT } \geq 20,000 / \mathrm{mm}^{3}\right. \\
\left.\text { and PLT }<50,000 / \mathrm{mm}^{3}\right)\end{array}$ & $\begin{array}{c}\text { Group } 3 \\
\text { (PLT }<50,000 / \mathrm{mm}^{3} \\
\text { elevated } \\
\text { to } \geq 50,000 / \mathrm{mm}^{3} \\
\text { before surgery) }\end{array}$ & \multirow[t]{2}{*}{$\begin{array}{c}\text { Group } 4 \\
\left(P L T \geq 50,000 / \mathrm{mm}^{3}\right)\end{array}$} & \multirow[t]{2}{*}{$P$-value } \\
\hline & \multicolumn{2}{|c|}{ Low PLT group } & High PLT group & & \\
\hline \multirow{2}{*}{$\begin{array}{l}\text { Number of } \\
\text { patients }\end{array}$} & 9 & 46 & 114 & 25 & \\
\hline & \multicolumn{2}{|r|}{55} & \multicolumn{2}{|c|}{139} & - \\
\hline \multirow[t]{2}{*}{ Male/female } & $5 / 4$ & $23 / 23$ & $41 / 73$ & $5 / 20$ & \\
\hline & \multicolumn{2}{|c|}{$28 / 27$} & \multicolumn{2}{|c|}{ 46/93 } & 0.21 \\
\hline \multirow[t]{2}{*}{ Age [years] } & $43.75 \pm 16.44$ & $35.96 \pm 15.73$ & $36.82 \pm 16.23$ & $39.45 \pm 16.77$ & \\
\hline & \multicolumn{2}{|c|}{$40.29 \pm 17.35$} & \multicolumn{2}{|c|}{$39.98 \pm 16.5$} & 0.91 \\
\hline \multirow[t]{2}{*}{ PLT } & $7.5 \pm 7.93$ & $40.82 \pm 17.53$ & $130 \pm 63.16$ & $108.4 \pm 57.32$ & \\
\hline & \multicolumn{2}{|c|}{$32.1 \pm 13.64$} & \multicolumn{2}{|c|}{$118.1 \pm 59.8$} & - \\
\hline \multirow{2}{*}{$\begin{array}{l}\text { Preoperative } \\
\text { need for } \\
\text { glucocorticoids* }\end{array}$} & 9 & 41 & 110 & 22 & \\
\hline & & 50 & & & 0.29 \\
\hline \multirow{2}{*}{$\begin{array}{l}\text { Preoperative } \\
\text { need for } \\
\text { immunoglobulins* }\end{array}$} & 1 & 7 & 18 & 1 & \\
\hline & & 8 & & & 0.87 \\
\hline \multirow{2}{*}{$\begin{array}{l}\text { Preoperative } \\
\text { need for PLT } \\
\text { transfusions* }\end{array}$} & 5 & 8 & 1 & 0 & \\
\hline & & 13 & & & $<0.001$ \\
\hline
\end{tabular}


were considered statistically significant when the $p$-value was $<0.05$.

\section{Results}

Patients with low PLT preoperatively more often required perioperative platelet transfusions (13 vs. 1 , $p<0.001)$. The mean operative time in the low PLT group and high PLT group was $90 \pm 42.1 \mathrm{~min}$ and $95 \pm 45 \mathrm{~min}$, respectively. There were no statistically significant differences in this parameter between groups ( $p=0.59$, Figure 2 ).

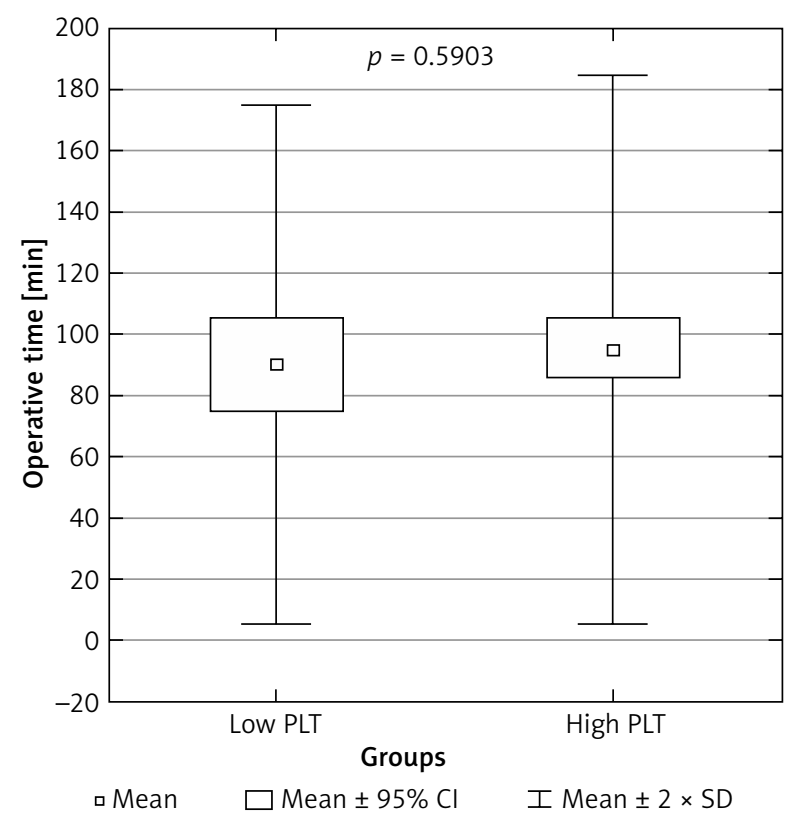

Figure 2. Comparison of the operative time in low PLT group and high PLT group
Intraoperative blood loss was $144 \pm 226.1 \mathrm{ml}$ in the low PLT group and $83 \pm 161.24 \mathrm{ml}$ in the high PLT group - this difference was not statistically significant ( $p=0.23$, Figure 3 ).

Complications occurred in 5 (9.09\%) patients in the low PLT group and 16 (11.51\%) in the high PLT group $(p=0.67)$. Characteristics of the complications are presented in Table II. There were no conversions in the group with lower PLT, while 2 patients in the group with higher PLT had to be converted to open surgery $(p=0.38)$. The reasons for conver-

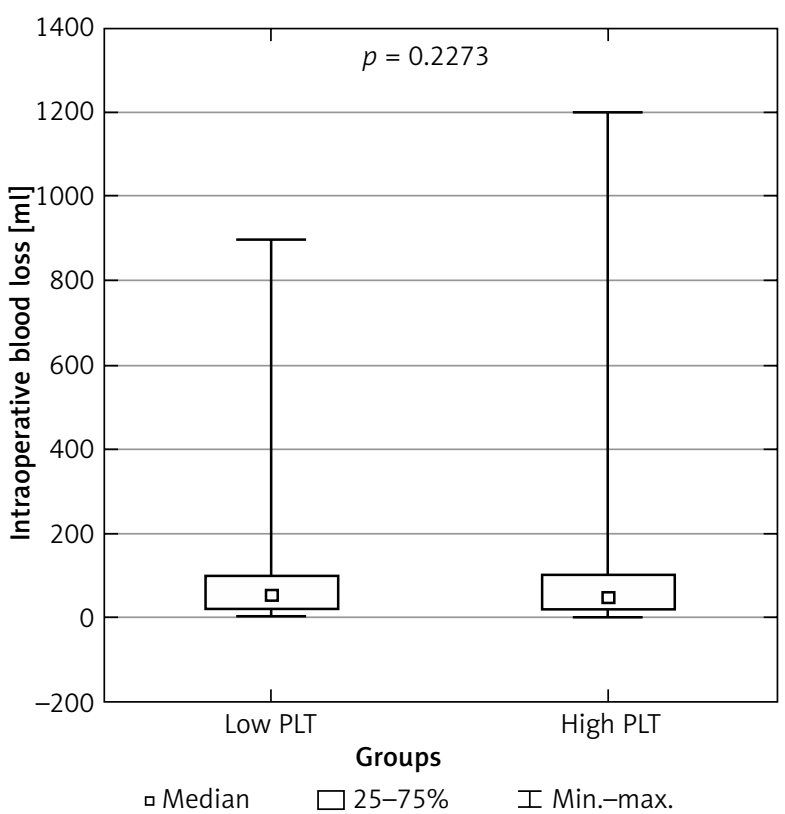

Figure 3. Intraoperative blood loss in low PLT group and high PLT group

Table II. Total complications in both groups according to Clavien-Dindo classification

\begin{tabular}{|lccc|}
\hline $\begin{array}{l}\text { Clavien-Dindo } \\
\text { grade }\end{array}$ & Complication & Low PLT group $(n=55)$ & High PLT group $(n=139)$ \\
\hline $\mathrm{I}$ & Seroma - fluid collection & 0 & $3(2.16 \%)$ \\
\hline $\mathrm{II}$ & Pneumonia & $1(1.81 \%)$ & $5(3.6 \%)$ \\
\hline $\mathrm{III} \mathrm{a}$ & Hematoma requiring subcutaneous drainage & $1(1.81 \%)$ & 0 \\
\hline & Abscess - required reoperation & $1(1.81 \%)$ & $2(1.44 \%)$ \\
\cline { 2 - 4 } & Acute pancreatitis - required reoperation & $1(1.81 \%)$ & 0 \\
\cline { 2 - 4 } & Bleeding - required reoperation & $1(1.81 \%)$ & $3(2.16 \%)$ \\
\hline IV b & Stomach perforation - required reoperation & 0 & $1(0.72 \%)$ \\
\hline $\mathrm{V}$ & Abscess - required reoperation & 0 & $1(0.72 \%)$ \\
\hline Total & Pulmonary embolism & 0 & $1(0.72 \%)$ \\
\hline
\end{tabular}


Table III. Summary of findings

\begin{tabular}{|lccc|}
\hline Parameter & Low PLT group & High PLT group & $P$-value \\
\hline Operative time [min] & $90 \pm 42.1$ & $95 \pm 45$ & 0.59 \\
\hline Intraoperative blood loss [ml] & $144 \pm 226.1$ & $83 \pm 161.24$ & 0.23 \\
\hline Complication rate & $5(9.09 \%)$ & $16(11.51 \%)$ & 0.67 \\
\hline Perioperative platelet transfusions & $13(23.6 \%)$ & $1(0.7 \%)$ & $<0.001$ \\
\hline
\end{tabular}

sion were uncontrolled bleeding in both cases: once during splenic hilum dissection and once during mobilization of the spleen. A summary of perioperative results is presented in Table III.

\section{Discussion}

We have confirmed that LS in patients with severe ITP and very low PLT is safe and feasible. It is not associated with a different course of surgical procedure or worse postoperative outcomes. In our opinion, the safety of the procedure in patients with end-stage thrombocytopenia might be affected by the strategy of perioperative management developed in our department. It is an important finding since ITP is one of the most common hematologic disorders affecting children and adults with the increasing destruction of platelets caused by the antiplatelet antibodies. Moreover, it is currently the most common indication for non-traumatic splenectomy. The percentage of patients with ITP who will eventually need splenectomy is reported to be, according to large series, as high as $20-45 \%$ [10-13].

According to recent a meta-analysis of more than 7,200 patients, LS for non-traumatic diseases of the spleen is superior to OS in terms of reduced blood loss and requirement for blood transfusion, lower morbidity and mortality rates and shorter length of hospital stay [14]. For these reasons, LS is now considered the gold standard for patients with ITP. Although there is a small percentage (7-22\%) of patients who do not respond to the treatment, it is still the best option in patients not eligible for conservative management [15].

Preoperative clinical symptoms of thrombocytopenia cause a great risk to patients and are sometimes regarded as a contraindication to surgery depriving patients of an effective method of treatment. Since the spleen in ITP is usually not enlarged, the greatest concern is low PLT that might lead to perioperative hemorrhage, difficulties with hemostasis requiring conversion and subsequent blood transfusions. On one hand it has been proven that LS significantly reduces the blood loss and need for platelet transfusion compared to open surgery [16]. On the other hand, coagulopathy is the absolute contraindication to laparoscopy in most guidelines and it should be corrected before surgery, which for obvious reasons is not possible in the majority of ITP patients [17]. Therefore, according to some authors, LS in low PLT should be contraindicated, whereas PLT higher than $50,000 / \mathrm{mm}^{3}$ is considered sufficient for effective hemostasis [18-20].

In our study, almost $30 \%$ of patients undergoing LS had PLT below $50,000 / \mathrm{mm}^{3}$ despite the most optimal preoperative management and adjustment of thrombocytopenia. Importantly, we have shown that low PLT is not associated with higher complication rates (complications occurred in 5 (9.09\%) patients in the low PLT group and in 16 (11.51\%) in the high PLT group). It was achieved with no difference in operative technique. For instance, despite very low PLT we still do not use drains, which according to previous observations do not bring any benefits and in some clinical situations may increase complication rates [21]. This confirms that LS is feasible and safe also in this, theoretically more dangerous, clinical situation. We did not find any statistically significant differences in either complication rates or their severity between groups. Patients with very low PLT did not present an increased rate of perioperative bleeding, which might be related to PLT level. In our study 1 patient from the low PLT group needed relaparoscopy because of bleeding, and 3 patients from the high PLT group. Two of them required conversion. These results are in line with other reports where complication rates were around 10\%, with lower morbidity in high-volume centers as noted by Montalvo et al. [22]. Although in our study we did not find any relationship between PLT and LS out- 
comes, there are previously published reports showing that severe thrombocytopenia might be a risk factor for increased morbidity. For instance, Keidar et al. showed that patients with severe thrombocytopenia had significantly more packed red cell transfusions and a much longer stay [23]. Both univariate and multivariate analysis of predictive factors by Duperier et al. confirmed that higher preoperative platelet count is associated with a successful response to LS [24].

We did not find any difference between low PLT level and greater difficulty of LS resulting in differences in operative time, increased blood loss or conversion rate. Having said that, the results may be biased because we did not take into account surgeons' experience. It is very likely that the most difficult cases were performed by more experienced surgeons in laparoscopic surgery of the spleen. On the other hand, the operative time in our study was lower than reported elsewhere. Chen et al. reported that from a group of 81 patients $9 \%$ had massive bleeding, 4 of them requiring conversion to open surgery [1]. Machado et al. found in their study that mean blood loss was 70 (range: 50-460) $\mathrm{ml}$ and operative time was 126 (range: 110-240) min [25]. In a systematic review Moris et al. found that LS can be performed with minimal blood loss $(30-60 \mathrm{ml})$, operative time was reported as $75-165 \mathrm{~min}$, and the conversion rate to OS was in the range $0-4 \%$ [26].

In order to establish optimal perioperative management, we developed a simple, easy to follow algorithm (Figure 1) for all surgeons dealing with patients with ITP. Every patient has to be referred by a consultant hematologist who confirms that LS is the optimal treatment of ITP at this stage. In all cases with PLT $\geq 50,000 \mathrm{~mm}^{3}$ no additional preoperative means of treatment are needed and patients can be operated on safely. In patients whose PLT assessed just before surgery is $<50,000 \mathrm{~mm}^{3}$, treatment modification is needed. They are referred back to the hematologist for further assessment and potential treatment modification. If hematologic intervention is successful (PLT $\geq 50,000 \mathrm{~mm}^{3}$ ) they can undergo LS. Patients with PLT between 20,000 and $50,000 / \mathrm{mm}^{3}$ despite intensive management and use of all therapeutic options may require platelet transfusions. Therefore 2 packs of platelets should be waiting in the blood bank to be ready for immediate transfusion in patients who do not respond to LS and sustain a low PLT count. Finally, the majority of patients with preoperative PLT $<20,000 \mathrm{~mm}^{2}$ will require intraoperative PLT transfusions. Therefore, we suggest considering starting transfusion of the first PLT unit at the beginning of the procedure and the next one once the splenic artery has been clipped. Generally the operative strategy is slightly different in those patients. The surgeon should identify, dissect and clip the splenic artery as early in the course of the operation as possible. This maneuver terminates PLT destruction in the spleen in most cases and allows for further dissection. With this strategy we were able to operate even on patients whose PLT level just before surgery was 0 . In our study, perioperative platelet transfusions were necessary only in 13 cases (in 6 cases during surgery and in the remaining 7 patients due to failure in PLT elevation postoperatively) in the low PLT group and 1 case in the group with high PLT (the patient did not respond to splenectomy). There are reports suggesting that continuous PLT transfusion may be successful in cases of serious uncontrolled bleeding with PLT below the measurable limit [27].

The limitations of the study are typical for a single retrospective analysis. In addition, we did not analyze other parameters that might influence the outcomes such as body mass index, previous abdominal surgery or the presence of an accessory spleen. Although they might have influenced the outcomes to some extent, none of them were identified as a reason for conversion or massive intraoperative bleeding. Moreover, due to the relatively small number of patients with very severe ITP (PLT $<20,000 \mathrm{~mm}^{3}$ ) we were not able to analyze them separately in order to achieve sufficient statistical power.

\section{Conclusions}

Our study confirms that LS is a safe and feasible surgical approach in patients with ITP regardless of the preoperative PLT. The procedure is not associated with a longer operative time, increased blood loss or conversion rate. Patients with low PLT are not at risk of increased morbidity. For these reasons, LS should be considered the gold standard in the surgical treatment of ITP. However, special precautions must be taken in the group of patients with severe ITP and extremely low PLT.

\section{Conflict of interest}

The authors declare no conflict of interest. 


\section{References}

1. Chen X, Peng B, Cai Y, et al. Laparoscopic splenectomy for patients with immune thrombocytopenia and very low platelet count: is platelet transfusion necessary? I Surg Res 2011; 170: e225-32.

2. Delaitre B, Maignien B. Splenectomy by the laparoscopic approach. Report of a case. Presse Med 1991; 20: 2263.

3. Zheng D, Huang CS, Huang SB, et al. Laparoscopic splenectomy for primary immune thrombocytopenia: current status and challenges. World J Gastrointest Endosc 2016; 8: 610-5.

4. Wu Z, Zhou J, Pankaj P, et al. Laparoscopic splenectomy for immune thrombocytopenia (ITP) patients with platelet counts lower than $1 \times 10^{9} /$ L. Int J Hematol 2011; 94: 533-8.

5. Bowers SP, Hunter JG. Contraindications to laparoscopy BT. In: The Sages Manual: Perioperative Care in Minimally Invasive Surgery. In: Whelan RL, Fleshman JW, Fowler DL (eds). Springer, New York, NY 2006; 25-32.

6. Feldman LS. Laparoscopic splenectomy: standardized approach. World J Surg 2011; 35: 1487-95.

7. Fisichella PM, Wong YM, Pappas SG, et al. Laparoscopic splenectomy: perioperative management, surgical technique, and results. J Gastrointest Surg 2014; 18: 404-10.

8. Pedziwiatr M, Matlok M, Major P, et al. Laparoscopic surgery of the spleen through single umbilical incision. Videosurgery Miniinv 2013; 8: 8-12.

9. von Elm E, Altman DG, Egger M, et al. The Strengthening the Reporting of Observational Studies in Epidemiology (STROBE) statement: guidelines for reporting observational studies. Int J Surg 2014; 12: 1495-9.

10. Pamuk GE, Pamuk ON, Baslar Z, et al. Overview of 321 patients with idiopathic thrombocytopenic purpura. Retrospective analysis of the clinical features and response to therapy. Ann Hematol 2002; 81: 436-40.

11. Stasi R, Stipa E, Masi M, et al. Long-term observation of 208 adults with chronic idiopathic thrombocytopenic purpura. Am J Med 1995; 98: 436-42.

12. Ben-Yehuda D, Gillis S, Eldor A. Clinical and therapeutic experience in 712 Israeli patients with idiopathic thrombocytopenic purpura. Israeli ITP Study Group. Acta Haematol 1994; 91: 1-6.

13. Pizzuto J, Ambriz R. Therapeutic experience on 934 adults with idiopathic thrombocytopenic purpura: multicentric trial of the cooperative Latin American group on hemostasis and thrombosis. Blood 1984; 64: 1179-83.

14. Chen J, Ma R, Yang S, et al. Perioperative outcomes of laparoscopic versus open splenectomy for nontraumatic diseases: a meta-analysis. Chin Med J (Engl) 2014; 127: 2504-10.

15. Rosen M, Brody F, Walsh RM, et al. Outcome of laparoscopic splenectomy based on hematologic indication. Surg Endosc 2002; 16: 272-9.

16. Vecchio R, Cacciola E, Lipari G, et al. Laparoscopic splenectomy reduces the need for platelet transfusion in patients with idiopathic thrombocytopenic purpura. JSLS 2005; 9: 415-8.

17. Hori Y; SAGES Guidelines Committee. Diagnostic laparoscopy guidelines: This guideline was prepared by the SAGES Guidelines Committee and reviewed and approved by the Board of Governors of the Society of American Gastrointestinal and
Endoscopic Surgeons (SAGES), November 2007. Surg Endosc 2008; 22: 1353-83.

18. Lozano-Salazar RR, Herrera MF, Vargas-Vorackova F, et al. Laparoscopic versus open splenectomy for immune thrombocytopenic purpura. Am J Surg 1998; 176: 366-9.

19. Walsh RM, Heniford BT, Brody F, et al. The ascendance of laparoscopic splenectomy. Am Surg 2001; 67: 48-53.

20. Katkhouda N, Grant SW, Mavor E, et al. Predictors of response after laparoscopic splenectomy for immune thrombocytopenic purpura. Surg Endosc 2001; 15: 484-8.

21. Major P, Matlok M, Pedziwiatr M, et al. Do we really need routine drainage after laparoscopic adrenalectomy and splenectomy? Videosurgery Miniinv 2012; 7: 33-9.

22. Montalvo J, Velazquez D, Pantoja JP, et al. Laparoscopic splenectomy for primary immune thrombocytopenia: clinical outcome and prognostic factors. J Laparoendosc Adv Surg Tech 2014; 24: 466-70.

23. Keidar A, Feldman M, Szold A. Analysis of outcome of laparoscopic splenectomy for idiopathic thrombocytopenic purpura by platelet count. Am J Hematol 2005; 80: 95-100.

24. Duperier T, Brody F, Felsher J, et al. Predictive factors for successful laparoscopic splenectomy in patients with immune thrombocytopenic purpura. Arch Surg 2004; 139: 61-6.

25. Machado NO, Al Kindy N, Chopra PJ. Laparoscopic splenectomy using LigaSure. JSLS 2010; 14: 547-52.

26. Moris D, Dimitriou N, Griniatsos J. Laparoscopic splenectomy for benign hematological disorders in adults: a systematic review. In Vivo 2017; 31: 291-302.

27. Yoneoka Y, Tokita K, Yamauchi J, et al. Successful management for spontaneous intracranial haemorrhage under critical thrombocytopenia (platelets $<1000 / \mathrm{mm}(3)$ ) due to adolescence-onset idiopathic thrombocytopenic purpura. Acta Neurochir (Wien) 2004; 146: 199-201.

Received: 16.12 .2017 , accepted: 19.02 .2018 